\title{
Vitamin K for Neonates Receiving Antibiotics
}

\author{
RHISHIKESH THAKRE \\ Consultant Neonatologist,Neo Clinic, Aurangabad.Maharashtra,India.rptdoc@gmail.com
}

$\mathrm{T}$ he role of vitamin $\mathrm{K}$ prophylaxis to prevent vitamin $\mathrm{K}$ deficiency bleeding (VKDB) in healthy newborns is well established. Secondary vitamin $\mathrm{K}$ deficiency has been reported in association with poor oral intake and use of broad-spectrum antibiotics in children and adults $[1,2]$. This phenomenon is under-recognized and underdiagnosed in newborns, and there is paucity of literature related to secondary vitamin $\mathrm{K}$ deficiency. The practice of weekly supplementation of vitamin $\mathrm{K}$ to newborns in hospital settings is thus 'empirical' and not well studied. In this issue of Indian Pediatrics, Sethi, et al. [3] report the prevalence of vitamin K deficiency in hospitalized newborns receiving antibiotics, and the role of routine vitamin $\mathrm{K}$ administration in prevention of coagulation abnormalities. The authors have compared the prevalence of vitamin $\mathrm{K}$ deficiency after intramuscular vitamin $\mathrm{K}$ on day 7 versus no vitamin $\mathrm{K}$ administration, in neonates with sepsis receiving prolonged $(>7 \mathrm{~d})$ antibiotic therapy, in a well-designed randomized controlled trial. Using PIVKA II (protein induced by vitamin $\mathrm{K}$ absence or antagonism) levels ( $>2 \mathrm{ng} / \mathrm{mL}$ ) as marker of vitamin $\mathrm{K}$ deficiency, they observed biochemical vitamin $\mathrm{K}$ deficiency in both groups with no evidence of difference in coagulation abnormalities or clinical bleeding between the two groups.

The authors report subclinical vitamin K deficiency in all neonates in whom PIVKA II levels were measured. Secondary vitamin $\mathrm{K}$ deficiency due to prolonged antibiotic therapy is postulated to be due to Nmethylthiotetrazole (NMTT) moiety containing antibiotics (e.g., cephalosporins), which are known to undermine vitamin $\mathrm{K}$ synthesis or its metabolism [4]. Antibiotics also cause replacement of normal gut flora resulting in deprivation of the natural source of vitamin K. Beyond antibiotics, several other factors contribute to vitamin $\mathrm{K}$ insufficiency - the severity of underlying illness, associated renal or hepatic dysfunction, poor oral intake, delayed feeding, malabsorption, and poor nutritional status. Cumulative intake of human milk is also known to regulate the vitamin $\mathrm{K}$ status in the first week [5]. The study [3] population had several of these risk factors predisposing to vitamin $\mathrm{K}$ deficiency.

The authors report very high PIVKA II levels at endline in both the groups, but with no difference in coagulation status. PIVKA II levels are normally absent from sera and its presence reflect partially carboxylated prothrombin in plasma suggesting vitamin $\mathrm{K}$ deficiency. Traditional coagulation tests (Prothrombin Time, Partial Thromboplastin Time) are neither sensitive nor specific for VKDB. The advantage of using PIVKA II is that it is detectable before any changes occur in the conventional coagulation tests, and helps identify early or subclinical vitamin K deficienccy. The high PIVKA II levels reported in the study are alarming. PIVKA II levels have been reported in the literature with wide variations. This could be due to different assay methods used, and their detection limits. Ethnicity or socioeconomic differences of the studied populations may also be contributory. The biggest drawback of using PIVKA II assay is lack of established normal values and standards. Hence, its usefulness as predictor of subclinical vitamin $\mathrm{K}$ deficiency remains to be demonstrated [6]. Increased PIVKA II may be observed in context other than vitamin K deficiency, and significant association between PIVKA II concentrations and the administration of antibiotics has been reported [7].

This study also reflects a weak clinical correlation between the biochemical indicators and abnormal bleeding in infants with vitamin $\mathrm{K}$ deficiency. This is paradoxical, as despite significant coagulation derangement observed biochemically, it did not translate to a bleeding phenotype. This study was not powered to detect VKDB, which is a rare event and sample size required to detect any difference in clinical bleeding will be much larger. Our understanding of the precipitating factors that trigger VKDB in an individual infant are less well understood.

It is worth noting that the study reported high prevalence of biochemical vitamin K deficiency in both the groups despite vitamin $\mathrm{K}$ administration. This raises 
questions about efficacy of the prophylactic dose of vitamin $\mathrm{K}$. It is a matter of speculation that multiple comorbid factors, maternal nutritional status or the antibiotic itself may directly or indirectly affect the action of vitamin $\mathrm{K}$. It needs to be investigated whether such infants may benefit from vitamin $\mathrm{K}$ at shorter intervals or higher dosages due to pharmacogenetic variations or polymorphism of genes for enzymes involved in vitamin $\mathrm{k}$ dependent coagulation factors [8].

The question remains regarding the clinical implications of this study [3]. Clinicians need to be aware that vitamin $\mathrm{K}$ deficiency is 'silent' problem in hospitalized newborn. Newborns on antibiotic therapy develop subclinical vitamin $\mathrm{K}$ deficiency, which is usually multifactorial and needs to be anticipated. PIVKA II is a biomarker reflecting low vitamin $\mathrm{K}$ levels, but it lacks normal reference range, and may not be directly associated with VKDB. A correct prophylaxis should prevent vitamin $\mathrm{K}$ deficiency, but the question of how best can we detect this deficiency early remains unresolved. There are several knowledge gaps in our understanding of the complex interaction between vitamin $\mathrm{K}$ and antibiotics. Further studies should attempt to investigate these complex interactions so that clinical decisions about vitamin $\mathrm{K}$ prophylaxis become evidence-based and not experiencebased, for experience is the ability to make the same mistake again and again with increasing confidence.
Funding: None; Competing interests: None stated.

\section{REFERENCES}

1. Aziz F, Patil P. Role of prophylactic vitamin $\mathrm{K}$ in preventing antibiotic induced hypoprothrombinemia. Indian J Pediatr. 2015;82:363-7.

2. Shevchuk YM, Conly JM. Antibiotic-associated hypoprothrombinemia: A review of prospective studies, 1966-1988. Rev Infect Dis. 1990;12:1109-26.

3. Sethi A, Jeeva Sankar M, Thukral A, Saxena R, Chaurasia $\mathrm{S}$, Agarwal A. Prophylactic vitamin K administration in neonates on prolonged antibiotic therapy: A randomized controlled trial. Indian Pediatr. 2019;56:463-7.

4. Lipsky JJ. Mechanism of the inhibition of the gammacarboxylation of glutamic acid by N-methylthiotetrazolecontaining antibiotics. Proc Natl Acad Sci USA. 1984;81:2893-7.

5. von Kries R, Becker A, Göbel U. Vitamin $\mathrm{K}$ in the newborn: Influence of nutritional factors on acarboxyprothrombin detectability and factor II and VII clotting activity. Eur J Pediatr. 1987;146:123-7.

6. Greer FR. Vitamin K the basics - what's new? Early Hum Dev. 2010;86(Suppl 1):43-7.

7. de Montalembert M, Lenoir G, Saint-Raymond A, Rey J, Lefrère JJ. Increased PIVKA-II concentrations in patients with cystic fibrosis. J Clin Pathol. 1992;45:180-1.

8. Lee SC, Ng SS, Oldenburg J, Chong PY, Rost S, Guo JY, et al. Interethnic variability of warfarin maintenance requirement is explained by VKORC1 genotype in an Asian population. Clin Pharmacol Ther. 2006;79:197-205. 